\title{
La perspectiva de género como una apertura conceptual y metodológica en salud pública
}

\author{
Gender approach as a conceptual and methodological opening \\ in public health
}

Dr. C. Pilar Serrano Gallardo

Universidad Autónoma de Madrid. Profesora de la Universidad Autónoma de Madrid. Madrid, España.

\section{RESUMEN}

La perspectiva de género propone y permite el análisis profundo de las relaciones sociales entre hombres y mujeres, con el fin de esclarecer las diferencias e inequidades en salud que están vinculadas al género. En el presente artículo se recorren aproximaciones teóricas y conceptuales que se han ido vinculando a la perspectiva de género para generar conocimiento, y entre las que se encuentran el marco conceptual de los determinantes de las desigualdades sociales en salud, la naturalización o esencialización, la masculinidad hegemónica en una sociedad patriarcal y el enfoque relacional de género. Por otra parte, se muestran la impronta de la perspectiva de género en cuestiones metodológicas: en la contextualización y fundamentación del problema de investigación, el diseño, en la selección de la población, la muestra y las variables, y en el análisis de los datos. La salud pública no se ha de conformar con contribuir a desvelar las desigualdades de género, sino que desde sus funciones de promocionar y mantener la salud de las poblaciones, ha de impulsar y colaborar activamente en el principio de igualdad entre mujeres y hombres en todos los niveles de la administración pública.

Palabras clave: perspectiva de género, salud pública, sesgo de género, modelos teóricos, metodología, desigualdades sociales en salud.

\section{ABSTRACT}

The gender perspective suggests and allows a deep analysis of social relations between men and women, in order to elucidate the differences and inequalities in 
gender-linked health. This paper went through theoretical and conceptual approaches that have been related to gender perspective to generate knowledge. Some of them are the conceptual framework of the determinants of social inequalities in health, the naturalization or essentialism, the hegemonic masculinity in a patriarchal society and the relational approach to gender. On the other hand, the impact of gender approach on methodological issues, for example on the conceptualization and justification of the research question, the design, on the selection of the population, the sample and variables and on data analysis, was shown. Public health should go beyond contributing to reveal gender inequalities; it should encourage and actively cooperate through its functions of promotion and preservation of the population's health to keep the principle of equality between women and men at all levels of the public management.

Key words: gender approach, public health, gender bias, theoretical models, methodology, social inequalities in health.

\section{NTRODUCCI ÓN}

Se puede definir género como el conjunto de creencias, rasgos personales, actitudes, sentimientos, valores, conductas y actividades que diferencian a hombres y mujeres a través de la construcción social. ${ }^{1}$ No es por tanto una característica biológica, sino un constructo analítico, que se fundamenta en la organización social de los sexos (la construcción social del sexo biológico). El género tiene un carácter cambiante atendiendo al contexto cultural y dinámico según el momento histórico. Desde esta concepción por tanto, las desigualdades de género son consideradas desigualdades sociales, y por consiguiente pueden ser modificadas.

También es preciso señalar que existen ideas preconcebidas tales como asumir que la situación de salud de hombres y mujeres son iguales, cuando en realidad no lo son, o dar por sentado que hay diferencias cuando lo que existen son similitudes, que llevan a cometer lo que se denomina sesgos de género. ${ }^{2}$

El enfoque o perspectiva de género, ${ }^{3,4}$ propone y permite el análisis profundo de las relaciones sociales entre hombres y mujeres, con el fin de esclarecer las diferencias e inequidades en salud que están vinculadas al género. Por otra parte, y probablemente formando parte de preconceptos erróneos sobre el enfoque de género, este no es solo apropiado para estudiar la salud de las mujeres, sino también la de los hombres. ${ }^{5-9}$

Castañeda Abascal, ${ }^{10}$ en su trabajo centrado en las reflexiones teóricas sobre las diferencias en salud atribuibles al género, sostiene como hipótesis central de la perspectiva de género, la influencia que la sociedad le confiere al hecho de ser mujer u hombre, y que lo que esto representa, puede provocar comportamientos y actitudes que determinan distintas maneras de exponerse al riesgo, y por lo tanto de determinar su salud.

La Conferencia Internacional sobre Población y Desarrollo, celebrada en 1994 en el Cairo, ${ }^{11}$ puede considerarse pionera en la propuesta de promover la equidad y la igualdad de los sexos y los derechos de la mujer. En los últimos 20 años, han sido muchas las iniciativas en la misma dirección, tanto desde los gobiernos, instituciones, 
colectivos, ..., pudiéndose resaltar la realizada por la Comisión sobre los Determinantes Sociales de la Salud (CDSS) (creada en 2005 por la Organización Mundial de la Salud (OMS) que emite un informe en el año 2009, cuyo principal finalidad es proponer medidas conducentes a disminuir las desigualdades sociales y mejorar la equidad sanitaria; y es importante resaltar que el género es considerado un eje fundamental de desigualdad, y desde ahí la CDSS plantea:

Unicamente será posible reducir las desigualdades sanitarias en una generación, si se mejora la vida de las mujeres, las jóvenes y las niñas, que representan aproximadamente la mitad de la población del mundo, y si se lucha contra las desigualdades entre los sexos. ${ }^{12}$

El propósito de este artículo es recorrer elementos conceptuales y metodológicos vinculados a la perspectiva o enfoque de género, los cuales están contribuyendo a aumentar y profundizar en el conocimiento sobre la salud de las personas, y por lo tanto constituyen una apertura para la salud pública.

\section{APERTURA CONCEPTUAL VI NCULADA A LA PERSPECTI VA DE GÉNERO}

El enfoque de género, desde un punto de vista epistemológico, ha ido generando conocimiento científico, construido reflexivamente en unas circunstancias históricas y sociológicas, y en conexión con otros marcos teóricos. A continuación se recorren algunas de estas aproximaciones teóricas y conceptuales, así como algunas de las contribuciones que se ha generado desde estas aproximaciones entroncadas con el enfoque de género.

\section{El marco conceptual de los determinantes de las desigualdades sociales en salud}

Ha sido desarrollado por la Comisión para Reducir las Desigualdades en Salud en España, y que se deriva de los modelos propuestos por Orielle Solar y Alec I rwin para la Comisión de Determinantes Sociales de la Salud de la OMS y por Vicenç Navarro, contiene factores estructurales (gobierno, políticas, mercado laboral, cultura y valores, ...) y factores intermedios (recursos materiales, factores psicosociales, conductuales y biológicos, servicios de salud) de las desigualdades en salud, pero además contempla, formando parte de los factores estructurales, unos ejes de desigualdad entre los que se encuentra el género, además de la etnia, la clase social, la edad y el territorio. Estos ejes determinan jerarquías de poder y se relacionan con la discriminación. ${ }^{13}$

Las desigualdades de género están en profunda interrelación con la clase social y la situación migratoria. ${ }^{14}$ Las mujeres suelen ubicarse en clases sociales más bajas y tener un nivel socioeconómico menor. Hay autores que plantean que la raza o la etnia ( vinculadas estrechamente a la condición de persona inmigrante) constituyen un antecedente y determinan la situación socioeconómica de la persona y la clase social en la que se encuentra una persona. ${ }^{15}$ La población inmigrante por índole económica, generalmente suele estar más empobrecida que la autóctona, si a esto se le añade que las mujeres suelen ubicarse en clases sociales más bajas, es probable que los efectos socioeconómicos sean más importantes en ellas. El idioma puede conllevar sesgo de selección en los estudios, y es más frecuente que las pérdidas o no estar dentro de los criterios de inclusión afecten más a mujeres que a hombres, porque por cuestiones laborales, es más probable que los hombres hablen el idioma del país receptor que las mujeres. ${ }^{16}$ También es importante contemplar las diferentes 
situaciones por las que una mujer migra, dado que unas lo hacen por razones económicas siendo ellas las cuidadoras principales de la familia, dejando incluso a sus hijos en su país (por ejemplo las mujeres centroamericanas), otras emigran solas en busca de mayor autonomía y desarrollo personal (mujeres de los países del Este de Europa), y otras fundamentalmente siguen a sus maridos teniendo como objetivo la unidad familiar (mujeres africanas). Esto genera escenarios muy distintos de como se va a desarrollar el proceso migratorio y la incorporación en el nuevo país. ${ }^{17,18}$

La relación entre los múltiples roles sociales desempeñados y la salud es un tema ampliamente estudiado en las mujeres. La literatura sobre el tema expone de manera consistente que el hecho de que la mujer lleve a cabo las tareas domésticas y el cuidado de los miembros de la familia (rol reproductivo) conjuntamente con trabajar fuera del hogar (rol productivo) afecta negativamente a su salud. ${ }^{19-21}$ Ahora bien, la relación entre tener múltiples roles y la salud no opera siempre en una única dirección por el complejo entramado de otros factores que intervienen en esta relación. El Estudio Longitudinal Australiano sobre la Salud de las Mujeres ${ }^{1}$ a lo largo de tres generaciones, ponen de relieve que el efecto de la ocupación sobre la salud puede ser para gratificar o para estresar, y que probablemente estén implicadas habilidades para afrontar estos roles que son las que modulan su efecto sobre la salud. La complejidad de cuidar (a niños, ancianos, esposos), con las dimensiones material, moral y emocional que conlleva, ${ }^{23}$ hace difícil que se pueda conocer la carga que supone para la persona que cuida a través de los enfoques clásicos que a lo sumo han contemplado la dimensión material.

La influencia del trabajo en la salud tiene también otras connotaciones culturales relacionadas con el papel protagonista que han tenido y siguen teniendo muchos hombres en el mantenimiento económico del hogar. Un estudio realizado con trabajadores temporales latinos indocumentados en Estados Unidos, refleja como este tipo de ocupaciones inestables y sin reconocimiento conlleva que se tiraran por tierra sus valores masculinos (ligados al mantenimiento de sus familias, que permanecen en sus países de origen), y genera que entren en crisis su identidad como hombres y como latinos, y esto a su vez ocasiona problemas de salud mental, abuso de drogas, violencia interpersonal. ${ }^{24}$

También las condiciones socioeconómicas del entorno en que se habita se relacionan con las desigualdades de género. Un trabajo realizado en Cuba en población de 65 años y más, pone de manifiesto que las diferencias de género (especialmente en labores del hogar, actitud ante la jubilación, maltrato y salud autopercibida) son mayores en zona con condiciones más desfavorables. ${ }^{25}$

Por otra parte, la calidad de vida relacionada con la salud y la salud autopercibida es peor en mujeres que en varones, ${ }^{26-28}$ y muestra interrelación con la clase social, la situación migratoria y la edad, si bien también se manejan hipótesis que postulan que el apoyo social (peor en las mujeres) puede ser uno de los determinantes que subyacen tras esas diferencias, ${ }^{29}$ así como la morbilidad diferencial (más problemas de salud en las mujeres). ${ }^{30}$

\section{La naturalización o esencialización}

Desde un enfoque feminista y antropológico se define como la percepción esencialista y biologicista del cuerpo humano (especialmente algunos cuerpos humanos como las mujeres, las personas de raza negra,...), lleva implícita la marginación social de estos colectivos por razones biológicas y anula la posibilidad de transformación. Desde esta naturalización, se entendería el diferente desempeño social de los hombres y de las mujeres (básicamente desde la capacidad reproductiva de las mujeres), 
favoreciéndose modelos explicativos que legitiman las desigualdades de género; cuando en realidad, son las desigualdades de género las que interpretan y ayudan a comprender las diferencias biológicas (de sexo) de una determinada manera y no al revés. Desde este planteamiento, no se tiene o se es de un determinado género, sino que el género se hace, se construye a través de prácticas corporales, sociales e institucionales. ${ }^{3,4}$

La revisión realizada por Emslie, ${ }^{31}$ pone de manifiesto que las mujeres con enfermedad coronaria se retrasan más que los hombres en buscar ayuda médica. Esto es parcialmente porque ellas creen que es una enfermedad de hombres. Un estudio cualitativo constata la diferencia de síntomas del infarto agudo de miocardio entre hombres y mujeres, mostrando estas un cortejo (conciencia gradual de los síntomas como disnea, ausencia de dolor torácico y demora en demandar atención médica) que no se ajusta a lo establecido tradicionalmente. ${ }^{32} Y$ es que, como refleja I sabel Calvo, las enfermedades cardiovasculares, y la cardiopatía isquémica en particular, se han investigado fundamentalmente en varones y extrapolado los resultados a las mujeres sin que haya evidencia científica sobre la validez para ellas. ${ }^{33}$

\section{La masculinidad hegemónica en una sociedad patriarcal}

Como plantea Scott-Samuel, ${ }^{34}$ esta masculinidad se refleja en patrones antisociales y no saludables de socialización. Este tipo de masculinidad hegemónica se caracteriza por atributos negativos (dureza, agresividad, exposición a riesgos, represión de emociones), positivos (fuerza, dar protección, decisión, coraje) y controvertidos (individualismo, competitividad, practicidad, racionalidad), y aboca a desigualdades de poder, que finalmente se traducen en desigualdades en salud. Verdonk y otros, ${ }^{35}$ tras realizar entrevistas a hombres jóvenes y de mediana edad en sus entornos de trabajo, observaron que el concepto de hombre ideal se equipara con ser ganador e invulnerable, y que el hombre real se prepara para competir y evita las acciones de cuidado dado que se asocian a lo femenino, y les resta masculinidad.

Un trabajo cualitativo realizado con varones muestra que la infertilidad es interpretada como una potencialidad reproductiva anulada, poniendo en riesgo su identidad como varón. Para completar la demanda de masculinidad construida desde el género, es preciso tener un hijo biológico, y eso justifica la ruptura con la pareja, si el objetivo no se podía alcanzar. ${ }^{36}$

Una investigación enmarcada en el uso de la terapia antirretroviral (TARGA), señala que a pesar de que el acceso es mayor en hombres que en mujeres, que podía vincularse con el hecho de favorecer al miembro de la familiar que traía el dinero a casa, los varones hacen un uso limitado de los servicios preventivos, lo que podía relacionarse con la ideología de la invulnerabilidad masculina. Por otra parte, las mujeres se sienten en la obligación de estar sexualmente disponibles para sus parejas, y les es más difícil decir " $n$ " o negociar el uso del condón. Por otro lado, para los hombres, tener relaciones extramaritales puede suponer una normalización en su salud física, formando parte del trabajo de construcción social de las relaciones. ${ }^{37}$ Además, a esto hay que añadirle que este problema de salud tiene una carga más estigmatizante en mujeres que en hombres, sobre todo porque sienten que serán rechazadas por sus parejas y sus entornos familiares y sociales, aun a pesar de que muchas de ellas hayan adquirido la infección en el marco de relaciones heterosexuales y monógamas. ${ }^{38}$

En estrecha conexión con la masculinidad hegemónica, se encuentra lo que Goicolea, ${ }^{39}$ define como sistema de machismo marianismo. El marianismo conceptualiza a las mujeres como pasivas, sumisas, modestas, con una idealización 
de la maternidad, y un repudio a la dirección de su sexualidad. En su trabajo refleja que el matrimonio no es un seguro para evitar los embarazos en la adolescencia y puede, por el contrario, darse situaciones de violencia y sexo no seguro, además de favorecer el abandono precoz de los estudios por parte de la adolescente.

\section{Enfoque relacional de género}

Con respecto a los enfoques y abordajes vinculados al estudio del género, surge en los últimos años una corriente crítica, vinculada al posestructuralismo, pero que incorpora la teoría relacional (el género como estructura social dinámica que se va constituyendo a través de los patrones relacionales entre hombres y mujeres), y que Connell, ${ }^{40}$ denomina enfoque relacional de género. Este enfoque rechaza el pensamiento categórico que define el género desde una clasificación dicotómica de cuerpos (masculino/femenino, cuerpo de hombre/cuerpo de mujer), y que se relaciona estrechamente con la esencialización ya definida anteriormente. Pero la autora va más allá, siendo también muy crítica con el planteamiento que se realiza desde la CDSS de la OMS, dado que solo contempla las relaciones de género desde el poder de los hombres sobre las mujeres y además no traza las relaciones de género en un ámbito transnacional, se queda fundamentalmente en un modelo de género categórico, en estadísticas de las diferencias categóricas, en una identificación tácita de género con las mujeres y una concepción pobre de los derechos. Para Connell la cultura médica moderna está en un pensamiento categórico y de esencialización.

Moreno Luna y otros, ${ }^{41}$ tras analizar el comportamiento que tendrían los médicos ante una persona con síntomas de ansiedad depresión, en función de si esta es varón o mujer, concluyen que, ante los mismos síntomas y situación clínica relacionada con cardiopatía, se pautaría ansiolítico al 77,2\% de las mujeres y al 58,2 \% de los hombres y se derivaría al cardiólogo al 9,5\% de las mujeres y al $24,5 \%$ de los hombres (o se daban diferencias significativas en esta actitud según fuera médica o médico).

Un análisis teórico sobre la atención al embarazo, parto y puerperio en la realidad cubana, revela que los prestadores de salud y la organización del servicio refuerzan la subordinación de las mujeres y la relación de dominación que se establece, dado que prevalece una concepción biologicista y medicalizada de la reproducción humana. ${ }^{42}$

Otro escenario en el que se pone de manifiesto este pensamiento categórico y de esencialización de los profesionales es el de los cuidados en el hogar. García-Calvente y otros, ${ }^{43}$ reflejan que dentro de las actitudes de los profesionales de la atención primaria hay una ausencia de cuestionamiento de la situación actual (las mujeres son las que cuidan y además lo hacen mejor) y una idealización de la familia como marco para idóneo para cuidar.

\section{APERTURA METODOLÓGI CA VI NCULADA A LA PERSPECTI VA DE GÉNERO}

El conocimiento científico que se ha venido desarrollando bajo la perspectiva de género ha ido planteando retos y encontrándose con hándicap, que han posibilitado contar a día de hoy con algunas lecciones aprendidas, pero también están identificadas cuestiones que siguen precisando de una profundización en aras de mejorar el proceso investigador.

En el inicio de todo proceso investigativo, y para delimitar el fenómeno de estudio, es necesario llevar a cabo una adecuada contextualización (antecedente y estado actual 
del problema) y fundamentación, y en este sentido, llevar a cabo una investigación bajo el enfoque de género plantea limitaciones. Por una parte, la literatura científica es aún pobre en marcos teóricos, lo que se traduce en un conocimiento deficitario del fenómeno de estudio, ${ }^{37}$ lo que tiene serias consecuencias en el planteamiento de la pregunta/objetivo/hipótesis de investigación, y en consecuencia en el método más apropiado para resolverla, ${ }^{44}$ Además, a día de hoy, no es fácil localizar la producción científica con perspectiva de género. Valga decir, que en el tesauro de Medline, el Medical Subject Heading (MeSH), solo hay un descriptor que contenga la palabra "gender", gender identity, pero que se define como la experiencia personal de sentirse hombre o mujer, sin aludir a la construcción social del género y aún menos, a un enfoque conceptual o metodológico. Ariño y otros, ${ }^{45}$ en su propuesta para evaluar la perspectiva de género en los proyectos de investigación, además de hacer una propuesta de algunos términos MeSH para la realización de búsquedas bibliográficas, recomiendan que en el caso que haya carencia de dicha perspectiva en la búsqueda realizada, ha de dejarse constancia de ello en la introducción. También, formando parte de la fundamentación o la finalidad de la investigación, señalan que se ha de dejar claro si va a contribuir a esclarecer y profundizar en las desigualdades de género, o incluso a su modificación.

En lo que respecta a los diseños de investigación, el enfoque de género ha supuesto una apertura tanto a los estudios cualitativos, como a los mixtos o diseños multimétodo, dado que permiten aprehender toda la complejidad de la construcción social de género. ${ }^{39,46,47}$

Probablemente las cuestiones relativas al método son las que se han plasmado más desde los inicios de la investigación con perspectiva de género. Calvente y otros, ${ }^{47}$ en su decálogo de recomendaciones para incorporar la perspectiva de género en investigación, señalan, como respecto a la población y muestra, que se ha de asegurar una adecuada representación de hombres y mujeres, así como de sus diversas experiencias y situaciones; Ariño y otros, ${ }^{45}$ además advierten de la posibilidad de cometer sesgo de selección relacionados con el diagnóstico, sobre la base de excluir a personas que presenten signos y síntomas diferentes a los tradicionalmente contemplados. Por otra parte, la literatura se hace eco especialmente del sesgo o confusión que puede suponer el no incluir variables que tengan sensibilidad de género, es decir, que permitan atraparlo, como son: la división sexual del trabajo, la satisfacción con las condiciones de trabajo, potencial de conciliación familiar que tiene el trabajo, conflictos maritales, vivencia del estado civil, satisfacción relacionada con el cuidado de otros, recursos disponibles para el trabajo reproductivo, entre otros. Baider y Bengel, ${ }^{48}$ por ejemplo, reflejan que el estar casado supone un factor protector para los hombres, dado que el matrimonio les provee de una gran estructura social y de contro, en cambio para las mujeres no es el estar casadas, sino el afrontamiento satisfactorio ante los conflictos maritales lo que se convierte en predictor de buena calidad de vida relacionada con la salud (las mujeres tienen el doble de probabilidad de desarrollar clínica depresiva ante la discordia marital). Un análisis de contenido, desde la perspectiva de género, sobre los cuestionarios de la última encuesta de salud española (ENS 2006), revela que se han producido mejoras con respecto a la ENS 2003, incorporado preguntas acerca del trabajo reproductivo y el tiempo que se le dedica, así como de factores de riesgos más vinculados a las mujeres. ${ }^{49}$

También dentro del método, y en lo que respecta al análisis de los datos, es posiblemente donde se ha hecho más énfasis, sobre todo dejando constancia de que no se puede usar "género"como sinónimo de "sexo", ${ }^{45-47}$ que los datos se han de presentar desagregados por sexo y que el análisis ha de permitir identificar diferencias, desigualdades y semejanzas en salud entre mujeres y hombres, en su contexto social, económico y cultural. ${ }^{8,9,45,47}$ En los modelos multivariantes se ha comprobado como la variable sexo genera múltiples términos de interacción. Por lo 
tanto, el llevar a cabo modelos multivariantes desagregados por sexo permite una mayor comprensión y riqueza interpretativa, dado que proporciona el escenario para que se exprese la complejidad del constructo de género, en lugar de ser controlado si la variable sexo es incorporada en los modelos, porque ser mujer no se reduce a no ser hombre, como ser negro no se reduce a no ser blanco. Por otra parte, como ya se ha dicho con anterioridad, el enfoque de género no es solo apropiado para estudiar la salud de las mujeres, porque los modelos desagregados por sexo, permiten conocer como interactúa por ejemplo el trabajo, de manera distinta en la salud de los hombres y de las mujeres. ${ }^{5-9,50}$ Es conveniente resaltar sin embargo qué cuestiones acerca de la magnitud y la significación de la diferencia entre varones y mujeres no son las fundamentales a investigar bajo este enfoque porque las medidas de asociación obtenidas en los diferentes modelos no son comparables entre ellas. ${ }^{5,7}$

\section{REFLEXI ONES FI NALES}

A lo largo del artículo se ha pretendido mostrar como la perspectiva o enfoque de género no se limita al estudio de una variable. El complejo entramado que se esconde detrás de las relaciones de género con la salud hacen necesario que se adopten enfoques y herramientas que permitan obtener un conocimiento profundo que contribuya a desarticular las desigualdades entre hombres y mujeres que afectan a su salud.

La Salud Pública no se ha de conformar con contribuir a desvelar las desigualdades de género, sino que desde sus funciones de promover, promocionar y mantener la salud de las poblaciones ha de impulsar y colaborar activamente en el mainstreaming de género, ${ }^{8}$ (transversalidad del principio de igualdad entre mujeres y hombres en todos los niveles de la administración pública), porque sólo así se podrá avanzar en la consecución de la igualdad entre mujeres y hombres e impulsar tendencias de cambio social.

\section{REFERENCI AS BI BLI OGRÁFICAS}

1. De Villota P. Globalización y Género. Madrid: Editorial Síntesis; 1999.

2. Ruíz MT, Verbrugge LM. A two view of gender bias in medicine. J Epidemiol Community Health. 1997; 51: 106-109

3. Esteban ML. El estudio de la Salud y el Género: las ventajas de un enfoque antropológico y feminista. Salud Colectiva. 2006;2:9-2.

4. Inhorn MC, Whittle L. Feminism meets the "new" " epidemiologies: foward an appraisal of antifeminist biases in epidemiological research on women's health. Soc Sci Med. 2001;53:553-67.

5. Kunkel SR, Atchley RC. Why gender matters: being female is not the same as not being male. Am J Prev Med. 1996; 12: 294-95.

6. Rohlfs I, Borrell C, Fonseca M do C. Género, desigualdades y salud pública: conocimientos y desconocimientos. Gac Sanit. 2000; 14(supl 3): S60-71. 
7. Ruíz-Cantero MT, Vives-Cases C, Artazcoz. A framework to analyse gender bias in epidemiological research. J Epidemiol Community Health. 2007;61(suppl II):ii46-ii53.

8. Ley orgánica para la igualdad efectiva de mujeres y hombres de 2007. Pub en BOE. 23 Marz 2007.

9. Borrel C, Artazcoz L (coordinador). Investigación en género y salud. Barcelona: SEE; 2007.

10. Castañeda Abascall I. Reflexiones teóricas sobre las diferencias en salud atribuibles al género. Rev Cubana Salud Pública [Internet]. 2007 [citado 6 Ago 2012 ]; 33(2). Disponible en:

http://scielo.sld.cu/scielo.php?script=sci_arttext\&pid=S0864$\underline{34662007000200011 \& \operatorname{lng}=\mathrm{es}}$

11. Naciones Unidas. Informe de la Conferencia Internacional sobre la Población y el Desarrollo. El Cairo, 5 a 13 de septiembre de 1994 [Internet]. Nueva York: NN. UU.; 2005 [citado 30 May 2012]. Disponible en: http://daccess-dds-ny.un.org/doc/UNDOC /GEN/N95/231/29/PDF/N9523129.pdf?OpenElement

12. Comisión sobre Determinantes Sociales de la Salud. OMS. Subsanar las desigualdades en una generación. Alcanzar la equidad sanitaria actuando sobre los determinantes sociales de la salud. Ginebra: OMS; 2009.

13. Comisión para Reducir las Desigualdades Sociales en Salud en España. Dirección General de Salud Pública y Sanidad Exterior Ministerio de Sanidad y Política Social. Avanzando hacia la equidad. Propuesta de Políticas e intervenciones para reducir las Desigualdades Sociales en Salud en España [Internet]. España: Comisión; 2010 [citado 29 May 2012]. Disponible en: http://www.mspsi.es/profesionales/saludPublica LprevPromocion/promocion/desigualdadSalud/docs/Propuesta_Politicas_Reducir_Desig ualdades.pdf

14. Borrel C, Artazcoz L. Las desigualdades de género en salud: retos para el futuro. Rev Esp Salud Pública. 2008; 82:245-9.

15. Oliver MN, Muntaner C. Race or class, or race and class? Researching health inequities among african americans: the imperative to understand social class. Ingt J Health Serv. 2005; 35:48598.

16. Pottie K, Ng E, Spitzer D, Mohammed A, Glazier R. Language Proficiency, Gender and Self-reported Health. An Analysis of the First Two Waves of the Longitudinal Survey of Immigrants to Canada. Can J Public Health. 2008;99(6):505-10.

17. Llacer A, Zunzunegui MV, del Amo J, Mazarrasa L, et Bolumar F. The contribution of a gender perspectiva to the understanding of migrants' health. J Epidemiol Community Health. 2007; 61(suppl 2):ii4-ii10.

18. Dyck I, Dossa P. Place, health and home. Gender and migration in the constitution of healthy space. Health Place. 2007; 13:691-701.

19. Cecilia Cruz A, Noriega M y Garduño MA. Trabajo remunerado, trabajo doméstico y salud. Las diferencias cualitativas y cuantitativas entre mujeres y varones. Cad Saúde Pública. 2003; 19:1120-38. 
20. Borrell C, Muntaner C, Benach J, Artazcoz L. Social class and self-reported health status among men and women: what is the role of work organization, household material standards and household labour? Soc Sci Med. 2004; 58: 1869-87.

21. Artazcoz L, Artieda L, Borrell, C, Cortès I, Benach J, García V. Combining job and family demands and being healthy: What are the differences between men and women? Eur J Public Health. 2004; 14:43-8.

22. Lee C, Powers JR.Number of Social Roles, Health, and Health-Being in three generations of australian Women. Int J Behav Med. 2002; 9: 195-215.

23. Martín Palomo T. Domesticar el trabajo: una reflexión a partir de los cuidados. Cuadernos de Relaciones Laborales. 2008;26(2):13-44.

24. Walter N, Bourgois $\mathrm{P}$, Loinaz HM. Masculinity and undocumented labor migration: injured latino day laborers in San Francisco. Soc Sci Med. 2004; 59: 1159-68.

25. Castañeda Abascal I, Pérez Labradal L, Sanabria Ramos G. Diferencias de género en las edades del ciclo vital de 65 años y más en el municipio Guanabacoa. Rev Cubana Med Gen Integr [Internet]. 2009 [citado 2012 Ago 06]; 25(4):54-64. Disponible en: http://scielo.sld.cu/scielo.php?script=sci_arttext\&pid=S0864 $\underline{21252009000400006 \& \operatorname{lng}=\mathrm{es}}$

26. Serrano-Gallardo P, Lizán-Tudela L, Díaz-Olalla J M, Otero A. Valores poblacionales de referencia de la versión española de las viñetas COOP/WONCA de calidad de vida en población adulta urbana. Med Clín. 2009;132(9):336-43.

27. Serrano-Gallardo P, Díaz-Olalla J M, Otero A, Bolumar F. Self-perceived health among migrant and native populations in Madrid: a gender perspective. Internat J Migration Health Social Car. 2009;5(4):43-57

28. Kunst AE, Bos V, Lahelma E, Bartley M, Lissau I, Regidor E et al. Trends in socioeconomic inequalities in self-assessed health in 10 European Countries. Int J Epidemiol. 2005;34:295-305.

29. Gallicchio L, Hoffman SC, Helzlsouer KJ. The relationship between gender, social support, and health-related quality of life in a community-based study in Washington County, Maryland. Qual Life Res. 2007; 16:77786,

30. Valls-LlobetC, Ojuel Solsona J, López Carrillo M, Fuentes Pujol M. Manifestación y medicalización del malestar en las mujeres. Observatorio de Salud de la Mujer del Ministerio de Sanidad y Consumo. El Programa de Formación de Formadores/as en Perspectiva de Género en Salud. Madrid: Ministerio de Sanidad y Consumo; 2007.

31. Emslie C. Women, men and coronary heart disease: a review of qualitative litarature. J Adv Nurs. 2005;51:382-95.

32. Albarran JW, Clarke BA, Crawford J. 'It was not chest pain really, I can't explain it!'. An exploratory study on the nature of symptoms experienced by women during their myocardial infarction. J Clin Nurs. 2007; 16: 1292301.

33. Calvo I. Situación en España de las enfermedades cardiovasculares en la mujer. Viur in Salut. 2010; 84:4-5. 
34. Scott-Samuel A. Patriarchy, masculinities and Health inequalities. Gac Sanit. 2009; 23(2): 159-60.

35. Verdonk $\mathrm{P}$, Seesing $\mathrm{H}$, de Rijk A. Doing masculinity, not doing health? a qualitative study among dutch male employees about health beliefs and workplace physical activity. BMC Public Health. 2010; 10:712.

36. Calero J L, Santana F. La solución ante la infertilidad: Representaciones de un grupo de varones atendidos por este padecimiento. Rev Cubana Endocrinol [Internet]. 2006 [citado 6 Ago 2012 ]; 17(2). Disponible en: http://scielo.sld.cu/scielo.php?script=sci arttext\&pid=S1561 $29532006000200002 \& \operatorname{lng}=$ es

37. Hirsch JS. Gender, sexuality, and antiretroviral therapy: using social science to enhance outcomes and inform secondary prevention strategies. AIDS. 2007;21 (suppl 5): S21S29.

38. Sandelowski M, Lambe C, Barroso J. Stigma in HIV-Positive women. J Nurs Scholarsh. 2004; 36: 122-8.

39. Goicolea I. Adolescent pregnancies in the Amazon Basin of Ecuador: a rights and gender approach to adolescents' sexual and reproductive health. Global Health Action. 2010; 3: 5280. doi 10.3402/gha.v3i0.5280.

40. Connell R. Gender, health and theory: Conceptualizing the issue, in local and world perspective. Social Sci Med. 2012: 74: 1675-83.

41. Moreno Luna ME, Clemente Lirola E, Piñeo Acín MJ, Martínez Matías MR, Alonso Gómez F, Rodríguez Alcalá FJ. Influencia del género en el manejo de cuadros ansioso/depresivos. Aten Primaria. 2000;26:554-8.

42. García J ordá D, Díaz Bernal Z. Perspectiva antropológica y de género en el análisis de la atención al embarazo, parto y puerperio. Rev Cubana Salud Pública [Internet]. 2010 [citado 6 Ago 2012];36(4): 330-6. Disponible en: http://scielo.sld.cu/scielo.php?script=sci_arttext\&pid=S0864 34662010000400007\&lng=es

43. García Calvente MM, del Río Lozano M, Castaño López E, Mateo Rodríguez I, Maroto Navarro G, Hidalgo Ruzzante N. Análisis de género de las percepciones y actitudes de los y las profesionales de atención primaria ante el cuidado informal. Gac Sanit. 2010;24(4): 293302.

44. Silva Ayçaguer LC. Deficiencias en la formulación de problemas de investigación en ciencias de la salud. Metas de Enferm. 2004; 7(2):51-5.

45. Ariño MD, Tomás C, Eguiluz M, Samitier ML, Oliveros T, Yago T. ¿Se puede evaluar la perspectiva de género en los proyectos de investigación? Gac Sanit. $2011 ; 25(2): 14650$.

46. Villela W, Monteiro S, Vargas E. A incorporação de novos temas e saberes nos estudos em saúde coletiva: o caso do uso da categoria gênero. Ciência Saúde Coletiva. 2009; 14(4): 997-1006. 
47. García Calvente MM, Jiménez Rodrigo ML, Martínez Morante E. Guía para la incorporar la perspectiva de género en la investigación en salud. Serie Monografías EASP № 48. Granada: Escuela Andaluza de Salud Pública; 2010.

48. Baider L, Bengel J. Cancer and the spouse: gender-related differences in dealing with health care and illness. Crit Rev Oncol Hematol. 2001;40:115-23.

49. Ruiz-Cantero MT, Carrasco-Portiño M y Artazcoz L. Logros y retos de género de la Encuesta Nacional de Salud de 2006: análisis de los cuestionarios de adultos y hogar. Gac Sanit. 2011;25(1):612.

50. Artazcoz L, Borrell C, Cortàs I et al. Occupational epidemiology and work related inequalities in health: a gender perspective for two complementary approaches to work and health research. J. Epidemiol Community Health. 2007;61: ii39-ii45.

Pilar Serrano Gallardo. Universidad Autónoma de Madrid. Madrid, España. Correo electrónico: pilar.serrano@uam.es 